\title{
Decentralized circadian clocks process thermal and photoperiodic cues in specific tissues
}

\section{AUTHOR(S):}

Shimizu, Hanako; Katayama, Kana; Koto, Tomoko; Torii, Kotaro; Araki, Takashi; Endo, Motomu

\section{CITATION:}

Shimizu, Hanako ...[et al]. Decentralized circadian clocks process

thermal and photoperiodic cues in specific tissues. Nature Plants 2015, 1: 15163.

\section{ISSUE DATE:}

2015-11-02

\section{URL:}

http://hdl.handle.net/2433/201387

\section{RIGHT:}

(c) 2015 Macmillan Publishers Limited.; The full-text file will be made open to the public on 2 May 2016 in accordance with publisher's 'Terms and Conditions for Self-Archiving'; この 論文は出版社版でありません。引用の際には出版社版をご確認ご利用ください。; This is not the published version. Please cite only the published version. 
Title:

Decentralized circadian clocks process thermal and photoperiodic cues in specific tissues

Authors: Hanako Shimizu ${ }^{1}$, Kana Katayama ${ }^{1}$, Tomoko Koto ${ }^{1}$, Kotaro Torii ${ }^{1}$, Takashi Araki ${ }^{1}$, \& Motomu Endo ${ }^{1,2 *}$

\section{Affiliations:}

1. Division of Integrated Life Science, Graduate School of Biostudies, Kyoto University, Sakyo, Kyoto 606-8501, Japan.

2. Japan Science and Technology Agency, PRESTO, 4-1-8 Honcho Kawaguchi, Saitama 332-0012, Japan.

*Correspondence to:

Motomu Endo

E-mail; moendo@lif.kyoto-u.ac.jp

Fax; +81-75-753-6470 
The circadian clock increases organisms' fitness by regulating physiological responses ${ }^{1}$. In mammals, the circadian clock in the suprachiasmatic nucleus (SCN) governs daily behavioral rhythms ${ }^{2}$. Similarly, in Arabidopsis, tissue-specific circadian clock functions have emerged, and the importance of the vasculature clock for photoperiodic flowering has been demonstrated ${ }^{3-5}$. However, it remains unclear if the vasculature clock regulates the majority of physiological responses, like the SCN in mammals, and if other environmental signals are also processed by the vasculature clock. Here, we studied the involvement of tissue-specific circadian clock regulation of flowering and cell elongation under different photoperiods and temperatures. We found that the circadian clock in vascular phloem companion cells is essential for photoperiodic flowering regulation; by contrast, the epidermis has a crucial impact on ambient temperature-dependent cell elongation. Thus, there are clear assignments of roles among circadian clocks in each tissue. Our results reveal that, unlike the more centralized circadian clock in mammals, the plant circadian clock is decentralized, where each tissue specifically processes individual environmental cues and regulates individual physiological responses. Our new conceptual framework will be a starting point for deciphering circadian clock functions in each tissue, which will lead to a better understanding of how circadian clock processing of environmental signals may be affected by ongoing climate change ${ }^{6}$.

In many organisms, the circadian clock plays essential roles that influence behavior, through transcriptional and hormonal regulation in response to environmental changes ${ }^{1,2}$. In mammals, for example, light and food availability signals entrain central and peripheral The circadian clock increases organisms' fitness by regulating physiological responses ${ }^{1}$. In mammals, the circadian clock in the suprachiasmatic nucleus (SCN) governs daily behavioral rhythms². Similarly, in Arabidopsis, tissue-specific circadian clock functions 
have emerged, and the importance of the vasculature clock for photoperiodic flowering has been demonstrated ${ }^{3-5}$. However, it remains unclear if the vasculature clock regulates the majority of physiological responses, like the SCN in mammals, and if other environmental signals are also processed by the vasculature clock. Here, we studied the involvement of tissue-specific circadian clock regulation of flowering and cell elongation under different photoperiods and temperatures. We found that the circadian clock in vascular phloem companion cells is essential for photoperiodic flowering regulation; by contrast, the epidermis has a crucial impact on ambient temperature-dependent cell elongation. Thus, there are clear assignments of roles among circadian clocks in each tissue. Our results reveal that, unlike the more centralized circadian clock in mammals, the plant circadian clock is decentralized, where each tissue specifically processes individual environmental cues and regulates individual physiological responses. Our new conceptual framework will be a starting point for deciphering circadian clock functions in each tissue, which will lead to a better understanding of how circadian clock processing of environmental signals may be affected by ongoing climate change ${ }^{6}$. In many organisms, the circadian clock plays essential roles that influence behavior, through transcriptional and hormonal regulation in response to environmental changes ${ }^{1,2}$. In mammals, for example, light and food availability signals entrain central and peripheral clocks $^{7,8}$. Although food anticipatory behavior is reported to be SCN independent ${ }^{7}$, the majority of peripheral clocks are regulated by the SCN, and those clocks form a more centralized network (Supplementary Figure 1$)^{9}$. In plants, by contrast, although the importance of thermal and photoperiodic signals for circadian clock entrainment is well understood $^{1,10}$, it is largely unknown which tissue processes these environmental signals and what kind of a network structure the plant circadian clock has. 
To decipher which tissues are responsible for processing thermal and photoperiodic signals, and regulation of flowering and cell elongation, we analyzed tissue-specific functions of the circadian clock. In Arabidopsis, the circadian clock consists of interlocking multi-feedback loops, and overexpression of a clock gene such as CIRCADIAN CLOCK ASSOCIATED 1 (CCA1) and TIMING OF CAB EXPRESSION 1 (TOC1) lead to perturbation of the endogenous clock system ${ }^{5,11,12}$. We recently established transgenic lines that overexpress CCA1-GFP under different organ/tissue-specific promoters, such as CCA1 (almost all organs/tissues), CHLOROPHYLL A/B BINDING PROTEIN 3 (CAB3, also called LHCB1.2, mesophyll), SUCROSE-PROTON SYMPORTER 2 (SUC2, vasculature companion cell), 3-KETOACYL-COA SYNTHASE 6 (CER6, epidermis), UNUSUAL FLORAL ORGANS (UFO, shoot apical meristem), and TERPENE SYNTHASE-LIKE SEQUENCE-1,8-CINEOLE (TPS-CIN, hypocotyl/root) in a wild-type background (referred to as CCA1::CCA1, CAB3::CCA1, SUC2::CCA1, CER6::CCA1, UFO::CCA1, and TPS-CIN::CCA1, respectively) ${ }^{5}$. Quantitative real-time PCR (qPCR) studies of these transgenic lines showed that CCA1-GFP was overexpressed under the control of each respective promoter, and rhythmic oscillations of a representative circadian clock gene was disrupted in the targeted tissue (Supplementary Figures 2 and 3). Using these lines, we demonstrated that circadian clock perturbation by the SUC2 promoter leads to a late-flowering phenotype under long-day condition ${ }^{5}$.

To further understand the regulation of vasculature-mediated flowering, we first examined if the other circadian clock in vasculature sub-tissues, such as (pro)cambium or xylem, also regulates flowering under long-day condition. To answer this question we produced two transgenic lines that express CCA1-GFP under HOMEOBOX GENE 8 (AtHB8, 
(pro)cambium) and IRREGULAR XYLEM 3 (IRX3, xylem) promoters (Supplementary Figure $2)^{13}$. Circadian clock oscillation was still observed in the vasculature of these lines, probably due to the small contribution of the targeted sub-tissue (Supplementary Figure 3a); nevertheless, circadian clock-regulated AtHB8 and IRX3 oscillations were at least perturbed in the targeted tissue, suggesting these lines function as expected (Supplementary Figure 3b). Consistent with the previous result, the CCA1::CCA1 and two independent SUC2::CCA1 lines showed late flowering. By contrast, the other lines, including AtHB8::CCA1 and IRX3::CCA1, flowered normally (Fig. 1a, b). Next, we examined if the circadian clock in phloem companion cells regulated flowering through the photoperiod pathway. A photoreceptor for photoperiodic flowering, cryptochrome2 (cry2), and florigen encoded FLOWERING LOCUS T (FT) function in phloem companion cells, and both are needed in the photoperiod pathway; mutants defective in these genes showed late flowering under long-day but not short-day condition ${ }^{14-17}$. Under short-day condition, all transgenic lines including CCA1::CCA1 and SUC2::CCA1s flowered normally, suggesting only the circadian clock in phloem companion cells regulates flowering through the canonical photoperiod pathway (Fig. 1c). A similar late-flowering phenotype under long-day was obtained when we tissue-specifically overexpressed TOC1-GFP (referred to as TOC1::TOC1, CAB3::TOC1, SUC2::TOC1, CER6::TOC1, UFO::TOC1, and TPS-CIN::TOC1) (Supplementary Figure 4). The observed distinct circadian phases among TOC1-GFP transgenic lines suggest that the circadian clocks in those tissues have unique periods under free-running condition. As TOC1 acts as a transcriptional repressor of $C C A 1^{18,19}$, CCA1 expression levels in the TOC-GFP transgenic lines should be low. On the other hand, CCA1 expression levels were high in the CCA1-GFP transgenic lines, implying a general causative factor for the late flowering is not so much the expression levels of clock genes but the arhythmicities of their expression levels, although there are admittedly a few anomalies in the data, probably due to variability of 
plants and some technical issues.

The circadian clock controls the time of flowering through the FT - CONSTANS (CO) module $^{20}$. To examine which step of the signaling pathway is crucially affected in these lines, we monitored the expression levels of FT and CO by qPCR. The lower FT expression levels of CCA1::CCA1 and SUC2::CCA1s in the evening were consistent with the late-flowering phenotype of these lines under long-day (Fig. 1a, b, d). By contrast, CCA1::CCA1 and SUC2::CCA1s did not have severe effects on the diel expression pattern and levels of $C O$, which is directly upstream of FT (Fig. 1d) ${ }^{20}$. Hence we concluded that the circadian clock in phloem companion cells regulates photoperiodic flowering mainly through the regulation of $\mathrm{CO}$ protein stability or FT expression rather than diel oscillation of $C O$. There is also a report that PHYTOCHROME INTERACTING FACTOR 4 (PIF4) can directly activate FT expression $^{21}$. However PIF4 expression was not affected in the SUC2::CCA1 (see below).

Photosynthesis is a clock-regulated process that affects flowering time ${ }^{22}$; therefore, we tested if CCA1::CCA1 and SUC2::CCA1s also regulate the photosynthetic efficiency of photosystem II. The $\Delta F / F m^{\prime}$ (Yield), $F v / F m$ and chlorophyll $a / b$ ratio were similar, suggesting the circadian clock in these tissues was not directly regulating photosynthetic efficiency (Supplementary Figure 5).

Regulation of hypocotyl elongation is another well-known response regulated by a circadian clock, and overexpression of CCA1 causes a long-hypocotyl phenotype ${ }^{11}$. To examine if the same circadian clock system that regulates flowering in phloem companion cells also regulates hypocotyl elongation, we measured hypocotyl length of 1-week-old seedlings grown under $12 \mathrm{~h}$ light $12 \mathrm{~h}$ dark (L/D) condition (Fig. 2a, b). Unlike flowering regulation, 
CCA1::CCA1 and two independent CER6::CCA1s caused a long-hypocotyl phenotype. The other lines, including SUC2::CCA1, showed a normal hypocotyl length that was similar to wild type.

We then examined if the long-hypocotyl phenotype was due to cell elongation or cell proliferation. Hypocotyl epidermal cell length and cell numbers were measured, and demonstrated that the numbers of cells were similar to each other, whereas the average epidermal cell length of the long axis was increased in CCA1::CCA1 and CER6::CCA1s, suggesting the epidermal clock regulates cell elongation, but not cell proliferation (Fig. 2c, d). To confirm if the effects of the epidermal clock are restricted to hypocotyl elongation, we measured petiole length under L/D. Consistent with hypocotyl regulation, petioles in CCA1::CCA1 and CER6::CCA1s also displayed a long-petiole phenotype (Fig. 2e, f). The importance of epidermal clock functions for hypocotyl and petiole cell elongation is in keeping with the proposed “epidermal-growth-control model” in ref. 23.

According to a recent study, PIF4 expression in the evening is gated by the circadian clock in hypocotyl length regulation ${ }^{24}$. PIF4 expression during the night period induces downstream gene expression, such as INDOLE-3-ACETIC ACID INDUCIBLE 29 (IAA29), HOMEOBOX PROTEIN 2 (AtHB2), PHYTOCHROME-INTERACTING FACTOR 3-LIKE 1 (PIL1) and XYLOGLUCAN ENDOTRANSGLYCOSYLASE 7 (XTR7) ${ }^{25}$. We measured expression levels of these genes under L/D. Consistent with the hypocotyl elongation results, PIF4 expression levels at night were higher in CCA1::CCA1 and CER6::CCA1s, and were similar to wild type in the other transgenic lines (Fig. 2g). Moreover, higher PIF4 expression at the night period resulted in higher expression of downstream genes at night and early morning when the hypocotyl was elongated (Fig. 2g and Supplementary Figure 6). These results indicate 
that the circadian clock in epidermis regulates hypocotyl length by inducing cell elongation through expression of PIF4, IAA29, AtHB2, PIL1, and XTR7 during the night period. Since IAA29, AtHB2, and PIL1 are not specifically expressed in epidermis but rather enriched in vasculature $^{25-27}$, inter-tissue signaling from the epidermal circadian clock to inner vasculature can be hypothesized. Since IAA29 is an auxin-responsive gene, horizontal auxin flow from epidermis to vasculature might be involved in the process.

Some reports have suggested that regulation of hypocotyl elongation by a circadian clock is also a photoperiodic response ${ }^{28}$. Hence we then checked if these long-hypocotyl and long-petiole phenotypes are regulated by day length. We additionally checked hypocotyl and petiole lengths under long-day and short-day, and revealed that CCA1::CCA1 and CER6::CCA1s showed longer hypocotyls and petioles than those of wild type, independent of day length, although hypocotyl lengths in wild type and all of the CCA1-GFP transgenic lines were also generally regulated in response to day length (Supplementary Figure 7). Therefore, we concluded that at least two different mechanisms are involved in the cell elongation, one of which we demonstrated here is an epidermal clock-dependent and photoperiod-independent pathway, and the other is a photoperiod or photosynthate-dependent pathway.

In plants, not only light but also temperature is crucial for circadian clock input ${ }^{1,10}$. Therefore we hypothesized that the decentralized and tissue-specific circadian clock systems can be controlled by different sensitivities to the environmental signals. We first measured hypocotyl length under L/D at different temperature conditions. Compared to wild type, CCA1::CCA1 and CER6::CCA1s showed longer hypocotyls over a range from $18^{\circ} \mathrm{C}$ to $27^{\circ} \mathrm{C}$ (moderate temperature), but showed similar hypocotyl lengths under lower or higher (more extreme) 
temperature conditions (Fig. 3a). To test the generality of the epidermal clock functions with regard to cell elongation, we then measured cotyledon area under $\mathrm{L} / \mathrm{D}$ at different temperatures. Although we could not see the difference under lower temperatures in the transgenic lines, the reduced cotyledon areas in CCA1::CCA1 and CER6::CCA1s were clearly observed when seedlings were grown under higher temperatures (Fig. 3a-c). Taken together, these data indicate that the epidermal clock regulates cell elongation in response to ambient temperature. We have not excluded the possibility that spatial expression patterns of the tissue-specific promoters we used here are temperature sensing; however, the different sensitivities to the higher ambient temperature of CER6::CCA1 hypocotyl elongation, as opposed to cotyledon expansion, argue against this possibility.

Lower and higher temperatures also affect flowering ${ }^{21,29}$. To determine which tissue-specific circadian clock is implicated in the ambient temperature-dependent flowering, we measured flowering time at $27^{\circ} \mathrm{C}$ under short-day. As expected, flowering time in wild type was accelerated compared to $22^{\circ} \mathrm{C}$ (Fig. 1c) ${ }^{21}$. Flowering time in SUC2::CCA1s was also accelerated, but these lines showed severe late flowering compared to wild type, suggesting the presence of another signaling pathway for flowering time regulation at high ambient temperature (Fig. 3d). We also measured flowering time at $16^{\circ} \mathrm{C}$ long-day (Supplementary Figure 8). Both CCA1::CCA1 and SUC2::CCA1 were insensitive to the lower temperature and showed similar flowering times compared to $22^{\circ} \mathrm{C}$, whereas the other lines showed later flowering times (Fig. 1b) ${ }^{29}$, suggesting thermal and photoperiod cues share the same signaling pathway. We concluded that the circadian clock in phloem companion cells also processes ambient temperature-dependent flowering regulation.

Our results indicate that circadian clocks in different tissues process specific environmental cues and regulate individual physiological responses (Fig. 4). Since thermal and 
photoperiodic signals are not always correlated with each other (Supplementary Figure 9), the independent regulation we demonstrated here is beneficial for plants' fine-tuned response to the environment. Consistent with these observations, several studies have mentioned that the plant circadian clock has unique gene expression profiles in specific tissues/organs and has different sensitivities to light and temperature ${ }^{3,4,30}$. Now these differences may be explained in terms of tissue-specific circadian clock systems. To our knowledge, this is the first identification of the epidermis as a temperature-signal processing site for cell elongation. It is important to note, however, that we have not excluded the possibility that temperature sensing may also occur in other tissues. Interestingly, we have demonstrated that the perception and processing of photoperiodic cues are performed in the same phloem companion cells ${ }^{5,15}$. By the same token, these observations imply that ambient temperature sensing and processing are performed together in epidermal cells.

In mammals, the more centralized circadian clock systems directly control the operation of the individual units that form the SCN. In this study, we have clearly demonstrated that plants have a decentralized and tissue-specific circadian clock system in which lower level components operate on local information to achieve whole plant physiological responses (Supplementary Figure 1). This decentralized system is also essentially different from the distributed system — also known as hierarchy-less system — because clear hierarchical coupling among mesophyll-vasculature and shoot-root have been demonstrated ${ }^{3,9}$ (Supplementary Figure 1). These conceptually unique differences first came to our attention through tissue-specific analyses, which will help to elucidate the biological functions of the circadian clock in each tissue and to integrate those tissue-specific circadian clock circuits into a whole system. These features of the plant circadian clock suggest potential applications for independent regulation of flowering and cell elongation in agriculture. To cope with 
global warming, agricultural chemical targeting of the epidermal circadian clock will become a promising strategy for regulating the growth of crops.

\section{References}

1. Doherty, C. J. \& Kay, S. A. Circadian control of global gene expression patterns. Annu Rev Genet. 44, 419-444 (2010).

2. Barclay, J. L., Tsang, A. H. \& Oster, H. Interaction of central and peripheral clocks in physiological regulation. Prog Brain Res. 199, 163-181 (2012).

3. James, A. B. et al. The circadian clock in Arabidopsis roots is a simplified slave version of the clock in shoots. Science 322, 1832-1835 (2008).

4. Yakir, E. et al. Cell autonomous and cell-type specific circadian rhythms in Arabidopsis.

Plant J. 68, 520-531 (2011).

5. Endo, M. et al. Tissue-specific clocks in Arabidopsis show asymmetric coupling. Nature 515, 419-422 (2014).

6. Wolkovich, E. M. et al. Warming experiments underpredict plant phenological responses to climate change. Nature 485, 494-497 (2012).

7. Tahara, Y. \& Shibata, S. Chronobiology and nutrition. Neuroscience 253, 78-88 (2013).

8. Eckel-Mahan, K. \& Sassone-Corsi, P. Metabolism and the circadian clock converge. Physiol Rev. 93, 107-135 (2013).

9. Baran, P. On Distributed Communications: I. Introduction to Distributed Communications Networks, RM-3420-PR August 1964, http://www.rand.org/publications/RM/baran.list.html. 10. Wigge, P. A. Ambient temperature signalling in plants. Curr Opin Plant Biol. 16, 661-666 (2013).

11. Wang, Z. Y. \& Tobin, E. M. Constitutive expression of the CIRCADIAN CLOCK 
ASSOCIATED 1 (CCA1) gene disrupts circadian rhythms and suppresses its own expression. Cell 93, 1207-1217 (1998).

12. Makino, S., Matsushika, A., Kojima, M., Yamashino, T. \& Mizuno, T. The APRR1/TOC1 quintet implicated in circadian rhythms of Arabidopsis thaliana: I. Characterization with APRR1-overexpressing plants. Plant Cell Physiol. 43, 58-69 (2002).

13. Fukuda, H. Signals that control plant vascular cell differentiation. Nat Rev Mol Cell Biol. 5, 379-391 (2004).

14. Endo, M., Mochizuki, N., Suzuki, T. \& Nagatani, A. CRYPTOCHROME2 in vascular bundles regulates flowering in Arabidopsis. Plant Cell 19, 84-93 (2007).

15. Takada, S. \& Goto, K. TERMINAL FLOWER2, an Arabidopsis homolog of HETEROCHROMATIN PROTEIN1, counteracts the activation of FLOWERING LOCUS T by CONSTANS in the vascular tissues of leaves to regulate flowering time. Plant Cell 15, 2856-2865 (2003).

16. Abe, M. et al. FD, a bZIP protein mediating signals from the floral pathway integrator FT at the shoot apex. Science 309, 1052-1056 (2005).

17. Wigge, P.A. et al. Integration of spatial and temporal information during floral induction in Arabidopsis. Science 309, 1056-1059 (2005).

18. Huang, W. et al. Mapping the core of the Arabidopsis circadian clock defines the network structure of the oscillator. Science 336, 75-79 (2012).

19. Gendron, J. M. et al. Arabidopsis circadian clock protein, TOC1, is a DNA-binding transcription factor. Proc Natl Acad Sci USA. 109, 3167-3172 (2012).

20. Imaizumi, T. Arabidopsis circadian clock and photoperiodism: time to think about location. Curr Opin Plant Biol. 13, 83-89 (2010).

21. Kumar, S. V. Transcription factor PIF4 controls the thermosensory activation of flowering. Nature 484, 242-245 (2012). 
22. King, R. W., Hisamatsu, T., Goldschmidt, E. E. \& Blundell, C. The nature of floral signals in Arabidopsis. I. Photosynthesis and a far-red photoresponse independently regulate flowering by increasing expression of FLOWERING LOCUS T (FT). J Exp Bot. 59, 3811-3820 (2008).

23. Kutschera, U. \& Niklas, K. J. The epidermal-growth-control theory of stem elongation: an old and a new perspective. J Plant Physiol. 164, 1395-1409 (2007).

24. Nusinow, D. A. et al. The ELF4-ELF3-LUX complex links the circadian clock to diurnal control of hypocotyl growth. Nature 475, 398-402 (2011).

25. Hornitschek, P. et al. Phytochrome interacting factors 4 and 5 control seedling growth in changing light conditions by directly controlling auxin signaling. Plant J. 71, 699-711 (2012). 26. Stokes, M. E., Chattopadhyay, A., Wilkins, O., Nambara, E. \& Campbell, M. M. Interplay between sucrose and folate modulates auxin signaling in Arabidopsis. Plant Physiol. 162, 1552-1565 (2013).

27. Turchi, L. et al. Arabidopsis HD-Zip II transcription factors control apical embryo development and meristem function. Development 140, 2118-2129 (2013).

28. Nomoto, Y., Kubozono, S., Yamashino, T., Nakamichi, N. \& Mizuno, T. Circadian clockand PIF4-controlled plant growth: a coincidence mechanism directly integrates a hormone signaling network into the photoperiodic control of plant architectures in Arabidopsis thaliana. Plant Cell Physiol. 53, 1950-1964 (2012).

29. Blázquez, M. A., Ahn, J.H. \& Weigel, D. A thermosensory pathway controlling flowering time in Arabidopsis thaliana. Nat Genet. 33, 168-171 (2003).

30. Michael, T. P., Salome, P. A. \& McClung, C. R. Two Arabidopsis circadian oscillators can be distinguished by differential temperature sensitivity. Proc Natl Acad Sci USA 100, 6878-6883 (2003).

31. Shimada, T. L., Shimada, T. \& Hara-Nishimura, I. A rapid and non-destructive screenable 
marker, FAST, for identifying transformed seeds of Arabidopsis thaliana. Plant J. 61, 519-528 (2010).

32. Porra, R. J., Thompson, W. A. \& Kriedeman, P. E. Determination of accurate extinction coefficients and simultaneous equations for assaying chlorophylls $a$ and $b$ extracted with four different solvents: verification of the concentration of chlorophyll standards by atomic absorption spectroscopy. Biochim. Biophys. Acta 975, 384-394 (1989).

Acknowledgements We thank M. Niwa, K. Ifuku, and Y.C. Brenda for technical assistance; Y. Kondo, S.L. Harmer and T. Imaizumi for helpful advice; J.A. Hejna for English proofreading. This work was supported by a JST PRESTO 14529738 (to M.E.), JSPS KAKENHI grants 25650097 (to M.E.), a Nakatani Foundation (to M.E.), a Mitsubishi Foundation (to M.E.), Grant-in-Aid for Scientific Research on Innovative Areas 87006029 (to M.E.), 26113510 (to M.E.) and 25113005 (to T.A.).

Author Contributions M.E. planned the experiments. H.S., T.K., K.T. and K.K. performed experiments. H.S. and M.E. wrote the manuscript. M.E. and T.A. supervised the project. All authors discussed the results and commented on the manuscript.

Author Information Reprints and permissions information is available at www.nature.com/reprints. The authors declare no competing financial interests. Readers are welcome to comment on the online version of this article at www.nature.com/nplants Correspondence and requests for materials should be addressed to M.E. (moendo@lif.kyoto-u.ac.jp). 


\section{Figure legends}

Figure 1 | Circadian clock functions in phloem companion cells are necessary for photoperiodic flowering

a, Flowering phenotype of one-month-old plants at $22^{\circ} \mathrm{C}$ under long-day. b, c, Total leaf number at flowering for CCA1::CCA1 and SUC2::CCA1s were increased compared with wild type at $22^{\circ} \mathrm{C}$ under long-day (b) but not under short-day (c) (error bars, s.d.; $\mathrm{n}=12$ ). $\mathbf{d}$, Diel expression of FT (left) and CO (right), measured by qPCR, in cotyledons of wild type and the other transgenic lines at $22^{\circ} \mathrm{C}$ under long-day (error bars, s.e.m.; $\mathrm{n}=3$ ). The highest values are set as 1 . Significant difference from the corresponding wild types at $* P<0.001$ by Dunnett's test.

Figure 2 | Circadian clock functions in epidermal cells are necessary for cell elongation a, b, Hypocotyl phenotype (a) and hypocotyl length (b) of 7-day-old seedlings at $22^{\circ} \mathrm{C}$ under L/D (error bars, s.d.; $\mathrm{n}=10$ ). Scale bar is $5 \mathrm{~mm}$. c, $\mathbf{d}$, The number of axially aligned hypocotyl epidermal cells (c) and their average cell lengths (d) of 7-day-old seedlings at $22^{\circ} \mathrm{C}$ under $\mathrm{L} / \mathrm{D}$ (error bars, s.d.; $n=10$ (c) and n=16 (d)). e, f, Petiole phenotype (e) and petiole length (f) of one-month-old plants at $22^{\circ} \mathrm{C}$ under L/D (error bars, s.d.; $\mathrm{n}=10$ ). Scale bars are $1 \mathrm{~cm}$. $\mathbf{g}$, Diel expression of PIF4 (left) and IAA29 (right) in whole seedlings. 7-day-old seedlings grown at $22^{\circ} \mathrm{C}$ under L/D were analyzed every four hours by qPCR (error bars, s.e.m.; $n=3$ ). The highest values are set as 1 . Significant difference from the corresponding wild types at ${ }^{*} P<0.001$ by Dunnett's test.

Figure 3 | An epidermal clock regulates cell elongation in response to ambient temperature 
a, Hypocotyl length (left) and cotyledon area (right) of 7-day-old seedlings for CCA1::CCA1 and CER6::CCA1s were affected at moderate ambient temperatures under L/D (error bars, s.d.; $\mathrm{n}=10) . \mathbf{b}, \mathbf{c}$, Cotyledon expansion phenotype at $22^{\circ} \mathrm{C}(\mathbf{b})$ and $31^{\circ} \mathrm{C}(\mathbf{c})$ under $\mathrm{L} / \mathrm{D}$. Scale bars are $5 \mathrm{~mm}$. d, Total leaf number at flowering was increased in SUC2::CCA1s compared to wild type at $27^{\circ} \mathrm{C}$ under short-day (error bars, s.d.; $n=10$ ). Significant difference from the corresponding wild types at $* P<0.001$ by Dunnett's test.

Figure 4 | A schematic drawing of the decentralized circadian clock system in Arabidopsis

a, A circadian clock in a phloem companion cell processes photoperiod signals perceived by cry $2^{14}$ and controls photoperiodic flowering through regulation of flowering hormone $(\mathrm{FT})^{16,17}$. b, The phloem companion cell clock also processes moderate ambient temperature signals perceived by an unknown thermoreceptor, and promotes flowering. c, A circadian clock in epidermis processes moderate ambient temperature signals and also controls cell elongation through regulation of the PIF4 and the other downstream gene expression in vasculature ${ }^{21,24 \text {, }}$ 25 .

\section{Methods}

\section{Plant material and growth conditions}

All wild type and transgenic lines were in the Arabidopsis thaliana ecotype Columbia-0 (Col-0) background. Seeds were surface-sterilized and sown on soil or $0.8 \%$ agar plates containing Murashige and Skoog medium with 0.5\% sucrose. Plants were grown under long-day (16 h light and $8 \mathrm{~h}$ dark, $42 \mu \mathrm{mol} \mathrm{m} \mathrm{m}^{-2} \mathrm{~s}^{-1}$ ), short-day (8 h light and $16 \mathrm{~h}$ dark, $84 \mu \mathrm{mol}$ $\left.\mathrm{m}^{-2} \mathrm{~s}^{-1}\right)$, and L/D (12 h light and $12 \mathrm{~h}$ dark, $56 \mu \mathrm{mol} \mathrm{m} \mathrm{m}^{-2} \mathrm{~s}^{-1}$ conditions at indicated 
temperatures. CCA1::CCA1, SUC2::CCA1, CAB3::CCA1, CER6::CCA1, UFO::CCA1 and TPS-CIN::CCA1 were established in a previous report ${ }^{5}$. Flowering times were scored by determining the number of total leaves when the first flower opened. Hypocotyl length and cotyledon area were measured by using ImageJ 1.48 (NIH).

\section{Tissue isolation}

Plants were grown under LD for 10 days at $22^{\circ} \mathrm{C}$. Cotyledons were collected at ZT0 and mesophyll, vasculature, and epidermis isolations were carried out as in ref. 5.

\section{Real-time PCR analysis}

Total RNA was extracted using an RNeasy Plant Mini Kit (Qiagen) and reverse-transcribed using a Transcriptor First Strand cDNA Synthesis Kit (Roche) according to the manufacturer's instructions. Real-time gene expression was analyzed with a CFX96 Real-Time PCR Detection System (Bio-Rad). The geometric mean of APA1 and IPP2 was used as a control ${ }^{5}$. Specific sequences for each primer pair were:

APA1-RT-F, 5'- TCCCAAGATCCAGAGAGGTC;

APA1-RT-R, 5'- CTCCAGAAGAGTATGTTCTGAAAG;

IPP2-RT- F, 5'- GTATGAGTTGCTTCTCCAGCAAAG;

IPP2-RT-R, 5'- GAGGATGGCTGCAACAAGTGT;

CCA1-RT-F, 5'- GACGAGGGTCGAATTGCCTT;

CCA1-RT-R, 5'- ACAGAGTCAAATGTTACAGGAAGAC;

TOC1-RT-F, 5'- GCCTCTTCGCACCAACGAGCT;

TOC1-RT-R, 5'- TCAGCAAGTCCTAGCATGCGTCT;

LUX-RT-F, 5'- GCTTCGGATAAGCTCTTCTCTTC;

LUX-RT-R, 5'- ATAAACTGGCATCTGCATCATCT; 
GFP-RT-F, 5'- GAGCTGAAGGGCATCGACTT;

GFP-RT-R, 5'- TTCTGCTTGTCGGCCATGAT;

FT-RT-F, 5'-CTAGCAACCCTCACCTCCGAGAATA;

FT-RT-R, 5'-CTGCCAAGCTGTCGAAACAATATAA;

CO-RT-F, 5'-TGCCGGTCAAACGCCTGCACCGT;

CO-RT-R, 5'-TGGCGGGAAGCAACGCGATTGGCA;

PIF4-RT-F, 5'-TAACGACCGTTGGACCTAGC;

PIF4-RT-R, 5'-TGCGTTCGGATTAAGCTTTT;

PIL1-RT-F, 5'-GGAAGCAAAACCCTTAGCATCAT;

PIL1-RT-R, 5'-TCCATATAATCTTCATCTTTTAATTTTGGTTTA;

IAA29-RT-F, 5'-ATCACCATCATTGCCCGTAT;

IAA29-RT-R, 5'-ATTGCCACACCATCCATCTT;

XTR7-RT-F, 5'-CGGCTTGCACAGCCTCTT;

XTR7-RT-R, 5'-TCGGTTGCCACTTGCAATT;

AtHB2-RT-F, 5'-GCTGAAGCAAACGGAGGTAG; and

AtHB2-RT-R, 5'-TTTGTAGCCGACGGTTCTCT;

The following thermal cycling profile was used,

$A P A 1,95^{\circ} \mathrm{C}$ for $10 \mathrm{~s}, \sim 40$ cycles of $95^{\circ} \mathrm{C}$ for $10 \mathrm{~s}, 66.6^{\circ} \mathrm{C}$ for $15 \mathrm{~s}$ and $72^{\circ} \mathrm{C}$ for $15 \mathrm{~s} ; I P P 2,95^{\circ} \mathrm{C}$ for $10 \mathrm{~s}, \sim 40$ cycles of $95^{\circ} \mathrm{C}$ for $10 \mathrm{~s}, 69.4^{\circ} \mathrm{C}$ for $15 \mathrm{~s}$ and $72^{\circ} \mathrm{C}$ for $15 \mathrm{~s} ; C C A 1,95^{\circ} \mathrm{C}$ for $10 \mathrm{~s}$, $\sim 40$ cycles of $95^{\circ} \mathrm{C}$ for $10 \mathrm{~s}, 67.1^{\circ} \mathrm{C}$ for $15 \mathrm{~s}$ and $72^{\circ} \mathrm{C}$ for $30 \mathrm{~s}$; TOC1, PIF4, PIL1, XTR7, $95^{\circ} \mathrm{C}$ for $10 \mathrm{~s}, \sim 40$ cycles of $95^{\circ} \mathrm{C}$ for $10 \mathrm{~s}, 60^{\circ} \mathrm{C}$ for $15 \mathrm{~s}$ and $72^{\circ} \mathrm{C}$ for $15 \mathrm{~s} ; G F P, 95^{\circ} \mathrm{C}$ for $10 \mathrm{~s}, \sim 40$ cycles of $95^{\circ} \mathrm{C}$ for $10 \mathrm{~s}, 62^{\circ} \mathrm{C}$ for $15 \mathrm{~s}$ and $72^{\circ} \mathrm{C}$ for $15 \mathrm{~s} ; L U X, 95^{\circ} \mathrm{C}$ for $10 \mathrm{~s}, \sim 40$ cycles of $95^{\circ} \mathrm{C}$ for $10 \mathrm{~s}, 65^{\circ} \mathrm{C}$ for $15 \mathrm{~s}$ and $72^{\circ} \mathrm{C}$ for $15 \mathrm{~s} ; \mathrm{FT}, 95^{\circ} \mathrm{C}$ for $10 \mathrm{~s}, \sim 40$ cycles of $95^{\circ} \mathrm{C}$ for $10 \mathrm{~s}, 61.9^{\circ} \mathrm{C}$ for $15 \mathrm{~s}$ and $72^{\circ} \mathrm{C}$ for $15 \mathrm{~s} ; \mathrm{CO}, 95^{\circ} \mathrm{C}$ for $10 \mathrm{~s}, \sim 40$ cycles of $95^{\circ} \mathrm{C}$ for $10 \mathrm{~s}, 65.4^{\circ} \mathrm{C}$ for $15 \mathrm{~s}$ and $72^{\circ} \mathrm{C}$ for $15 \mathrm{~s} ;$ IAA29, $95^{\circ} \mathrm{C}$ for $10 \mathrm{~s}, \sim 40$ cycles of $95^{\circ} \mathrm{C}$ for $10 \mathrm{~s}, 67.4^{\circ} \mathrm{C}$ for $15 \mathrm{~s}$ and $72^{\circ} \mathrm{C}$ for 
$15 \mathrm{~s}$; and $\mathrm{AtHB} 2,95^{\circ} \mathrm{C}$ for $10 \mathrm{~s}, \sim 40$ cycles of $95^{\circ} \mathrm{C}$ for $10 \mathrm{~s}, 67.6^{\circ} \mathrm{C}$ for $15 \mathrm{~s}$ and $72^{\circ} \mathrm{C}$ for $15 \mathrm{~s}$; Each sample was run in technical triplicate to reduce experimental errors. Error bars were calculated from the result of biological replicates (in most case biological triplicates). Data were analyzed by delta-delta-Ct method using CFX manager (Bio-Rad).

\section{Plasmid construction}

For AtHB8::CCA1-GFP and IRX3::CCA1-GFP constructs, the amplified fragment was digested with SalI and cloned into previously constructed $p E N T R 1 A / C C A 1-G F P^{5}$, and cloned into $\mathrm{pFAST}-\mathrm{R} 01^{31}$. The tissue-specific promoters were amplified by PCR using the following primers:

AtHB8-promoter-F, 5'-GTCGACCGGATAAACCAATTTTCAA;

AtHB8-promoter-R, 5'-GTCGACCTTTGATCCTCTCCGATCT;

IRX3-promoter-F, 5'-GTCGACAAAAATAAGTAAAAGATCT; and IRX3-promoter-R, 5'-GTCGACAGGGACGGCCGGAGATTAGCAG.

For Tissue-specific promoter driven TOC1-GFP lines, we have applied the same strategy as described in our previous report ${ }^{5}$.

\section{Measurement of photosynthetic efficiency of photosystem II.}

Ten-day-old seedlings were collected and immersed in N, N-dimethylformamide to extract chlorophyll. The chlorophyll-extracted liquids were measured for absorbance values at 647 and $664.5 \mathrm{~nm}$ using a spectrophotometer, and chlorophyll $a$ (chl $a$ ) and chlorophyll $b$ (chl $b$ ) contents were calculated according to the formula reported by Porra et $\mathrm{al}^{32}$. Chlorophyll fluorescence parameters were measured using a MINI-PAM (pulse-amplitude modulation) portable chlorophyll fluorometer (Walz, Germany). The maximum quantum yield $[\Delta \mathrm{F} / \mathrm{Fm} '$ 
(Yield)] and [Fv/Fm] were calculated based on the measured chlorophyll fluorescence. The leaves were exposed to low light $\left(120 \mu \mathrm{mol} \mathrm{m}^{-2} \mathrm{~s}^{-1}\right)$ for the measurement of all chlorophyll fluorescence parameters.

\section{Temperature and photoperiod data}

The monthly average temperature data were obtained from Japan meteorological agency (http://www.data.jma.go.jp) and Met office (http://www.metoffice.gov.uk). Average photoperiods were calculated based on sunrise and sunset time obtained from National astronomical observatory of japan (http://eco.mtk.nao.ac.jp).

\section{Accession numbers}

Sequence data from this article can be found in The Arabidopsis Information Resource (TAIR) databases under the following accession numbers: CCA1 (At2g46830), TOC1 (At5g61380), LUX/PCL1 (At3g46640), SUC2 (At1g22710), AtHB8 (At4g32880), IRX3 (At5g17420), FT (At1g65480), CO (At5g15840), CER6 (At1g68530), PIF4 (At2g43010), IAA29 (At4g32280), AtHB2 (At4g16780), PIL1 (At2g46970), XTR7 (At4g14130), CAB3 (At1g29910), UFO (At1g30950), TPS-CIN (At3g25820), APA1 (At1g11910) and IPP2 (At3g02780). 
Fig. 1
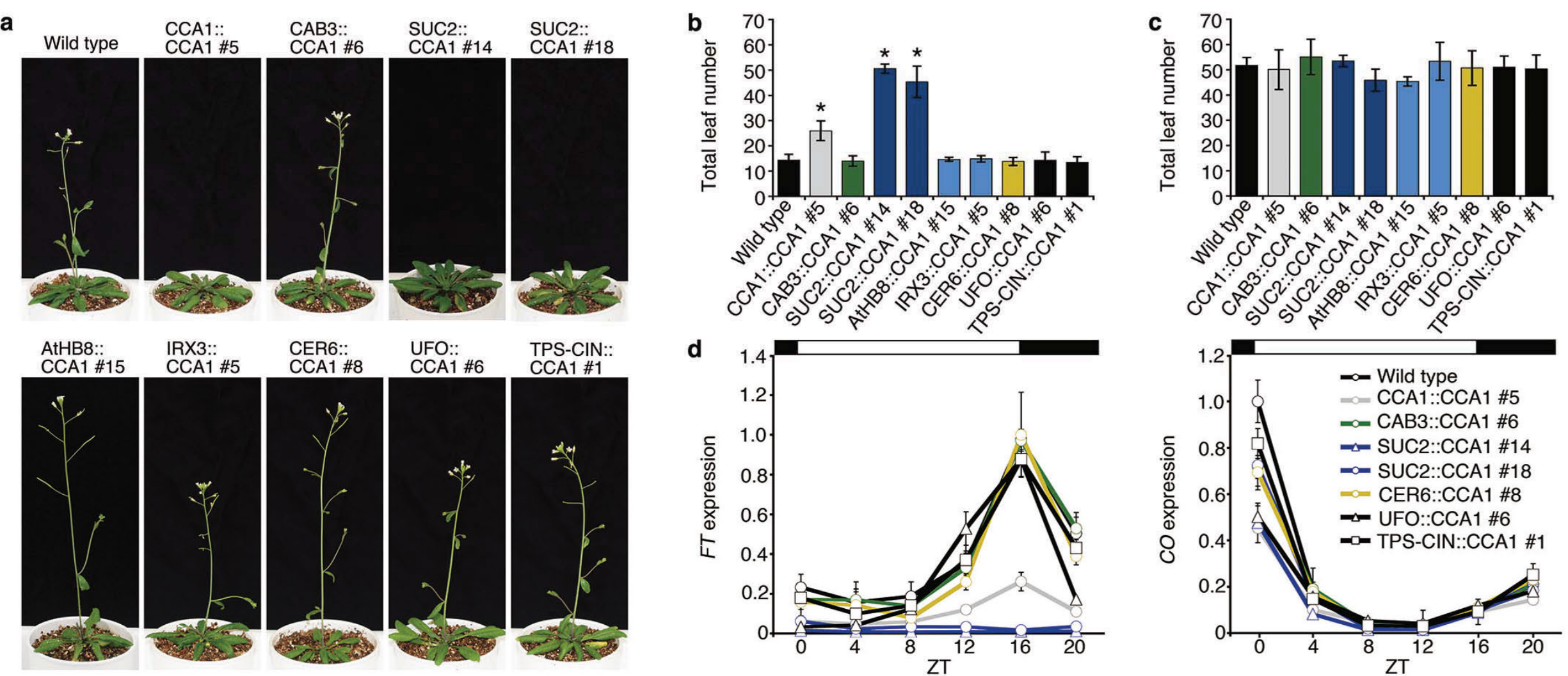
Fig. 2
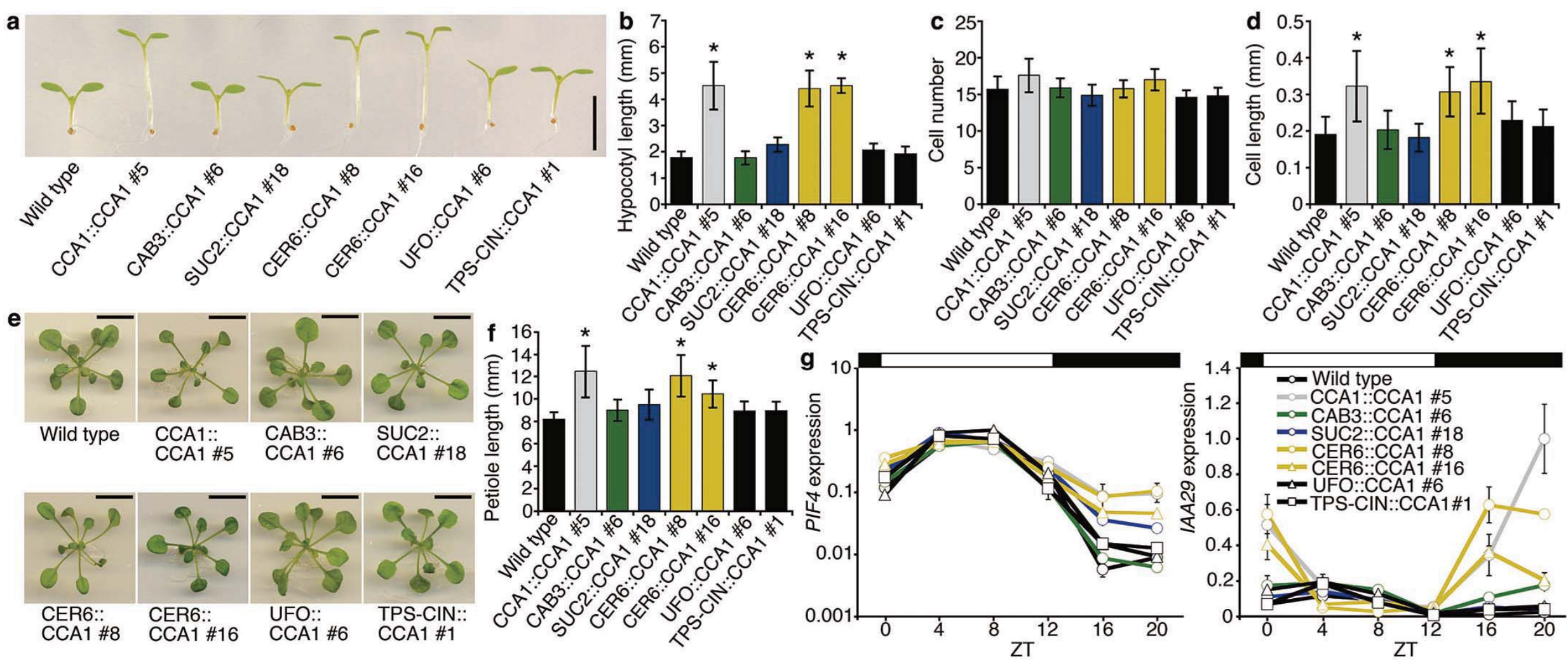
Fig. 3
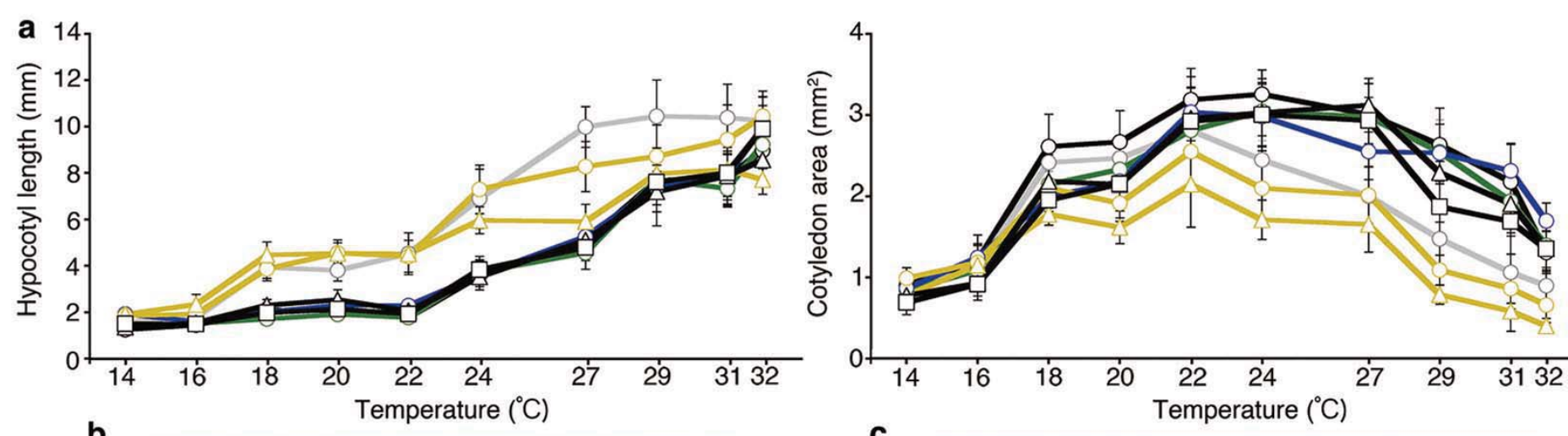

$\infty$ Wild type

CCA1...CCA1 \#5

- SUC2::CCA1

$=-\mathrm{CER} 6$. CCA $1 \# 8$

$\longrightarrow$ CER6::CCA1 \#16

- - TPS-CIN::CCA1\#1

d 70

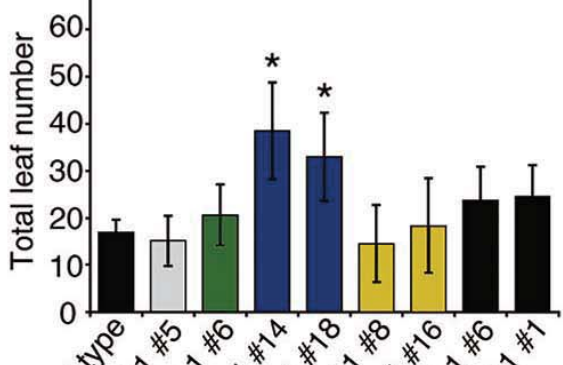

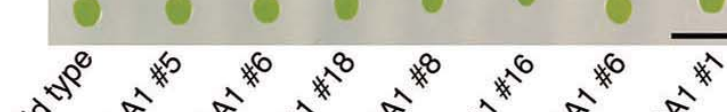

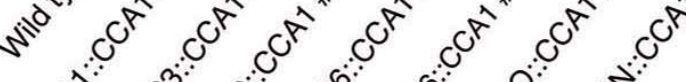

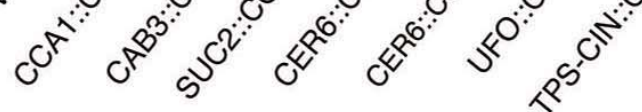

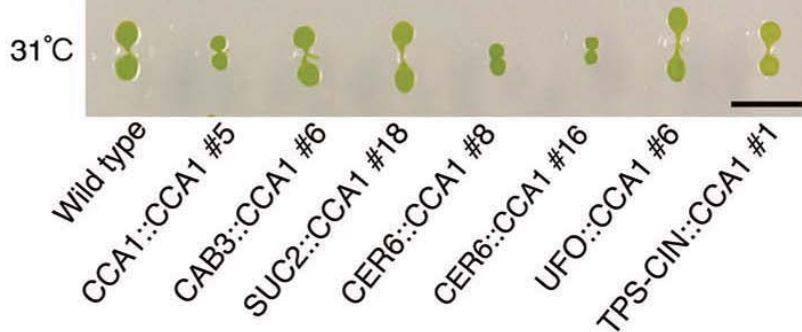

$00^{0} \times x^{10} \times x^{8} \times x^{6}$

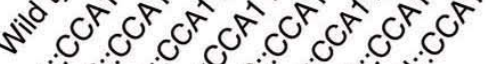

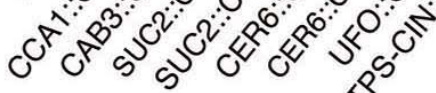




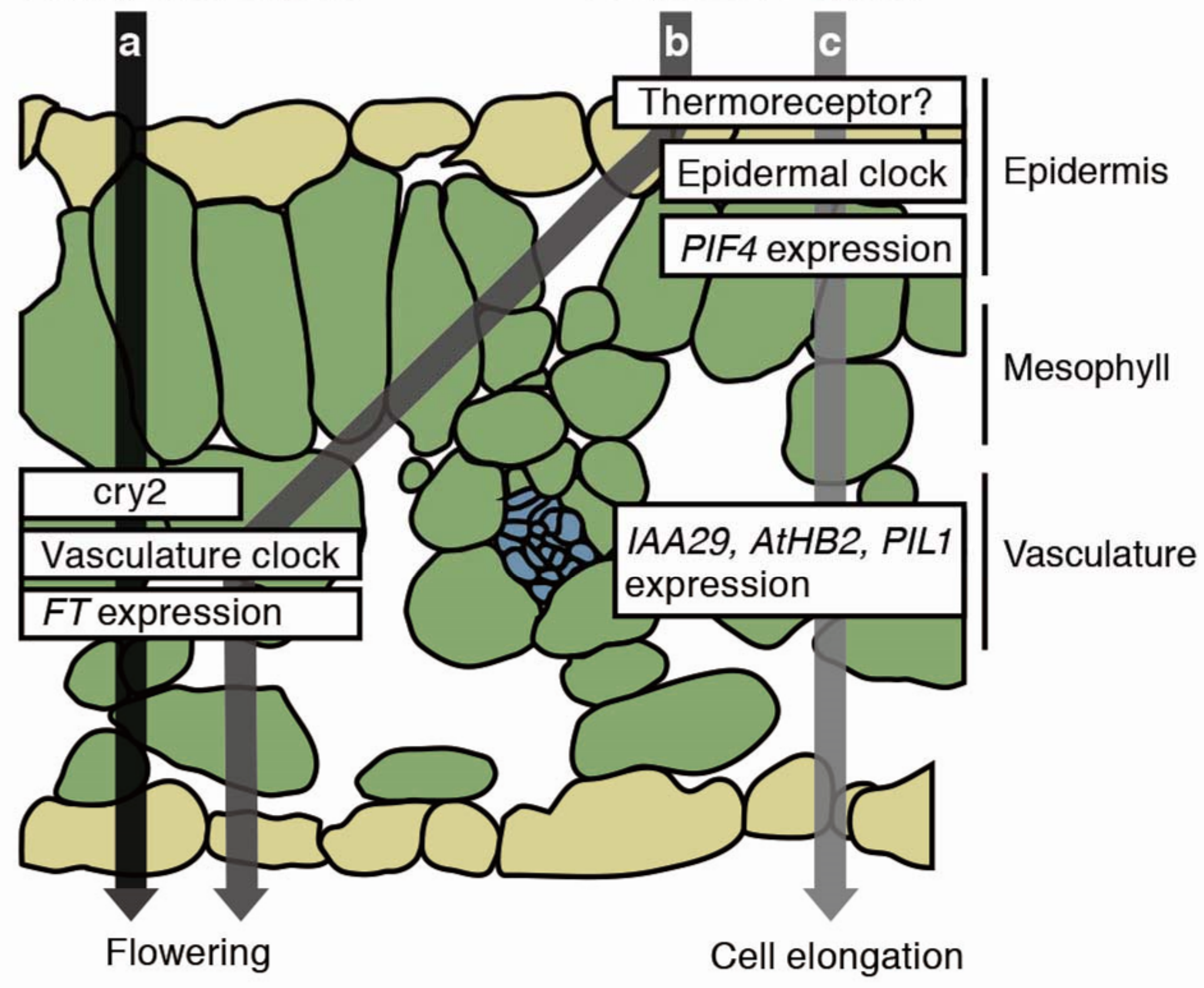



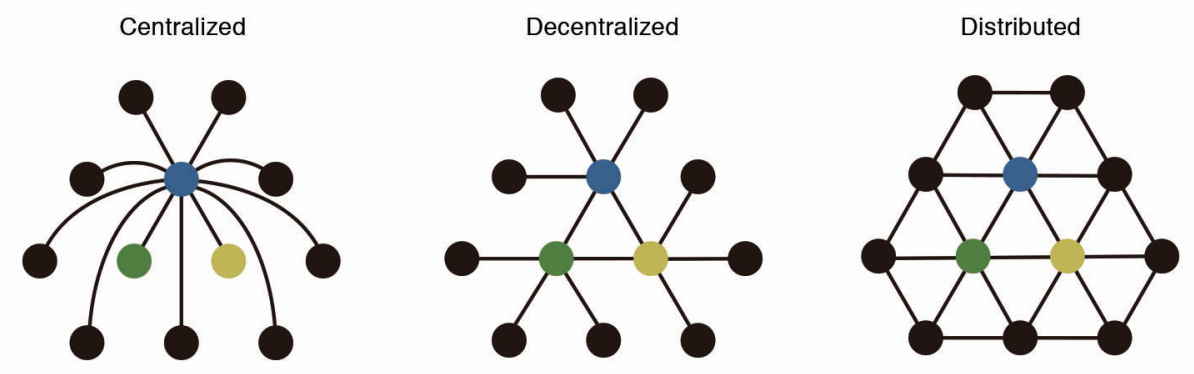

Supplementary Figure 1 I Centralized, de-centralized, and distributed networks

Schematic drawings of centralized, de-centralized, and distributed networks ${ }^{9}$. Centralized and de-centralized networks pertain to the hierarchical level at which decisions are made, whereas a distributed network does not have a specific node at which decisions are made. 
CCA1::CCA1 \#5
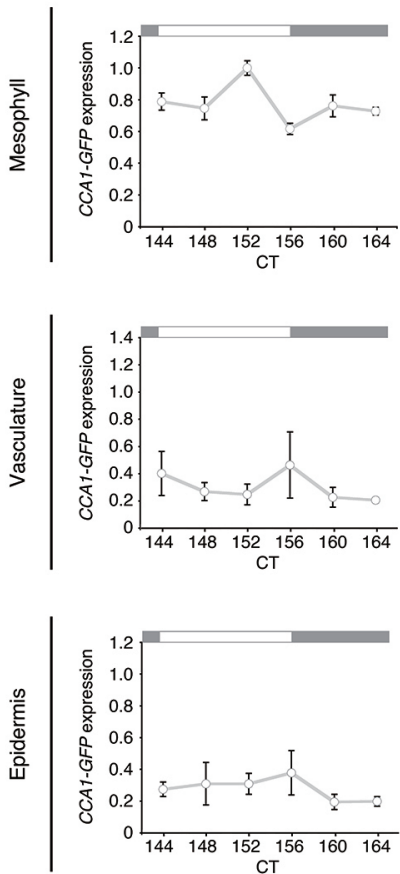

CER6::CCA1 \#8
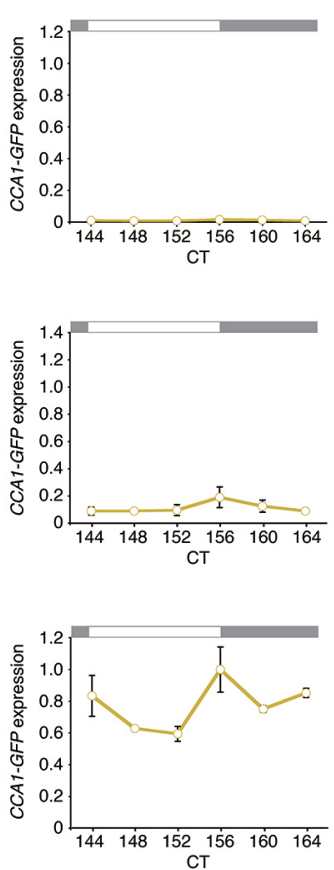

AtHB8::CCA1 \#15
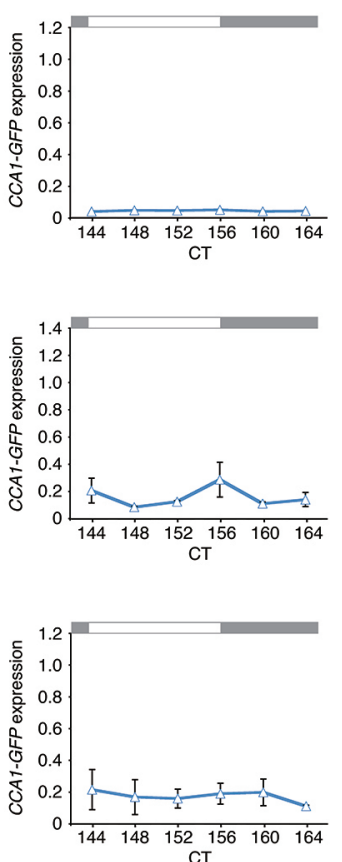

IRX3::CCA1 \#5
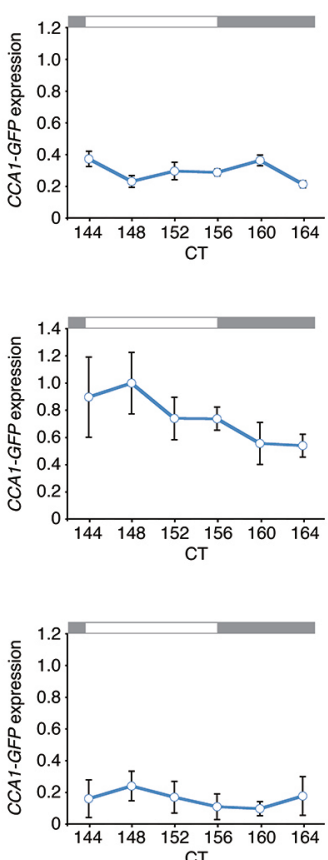

Supplementary Figure 2 I Tissue-specific expression of CCA1-GFP driven by tissue-specific promoters Expression levels of CCA1-GFP in each tissue of a cotyledon. Plants were grown under L/D for 5 days and then transferred into continuous light conditions for 6 days. Seedlings were separated into mesophyll, vasculature, and epidermis every $4 \mathrm{~h}$ on day 12 . For the CCA1-GFP detection, GFP expression was measured by qPCR and the geometric mean of $A P A 1$ and IPP2 was used as a control (error bars, s.e.m.; $\mathrm{n}=3$ ). CT, circadian time. The highest values are set as 1 . 

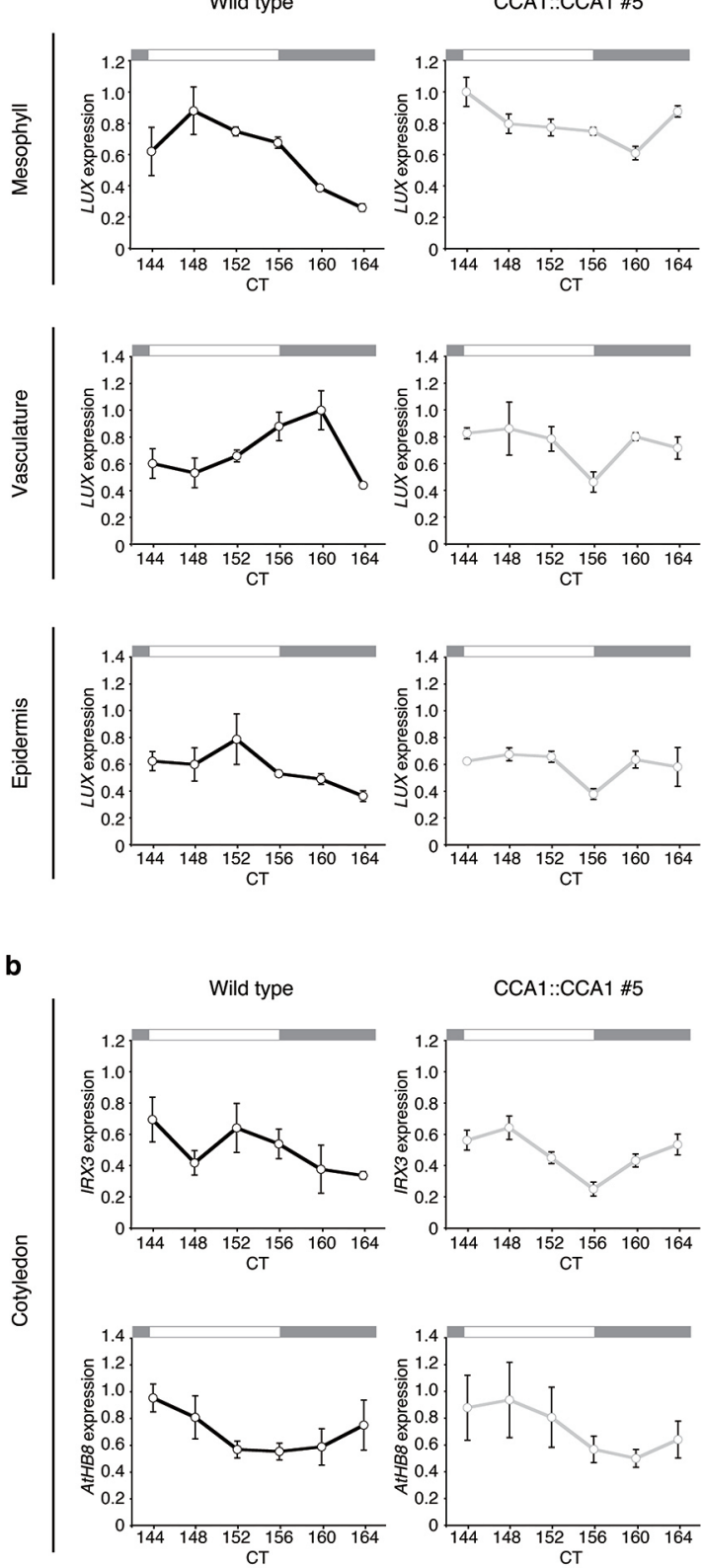
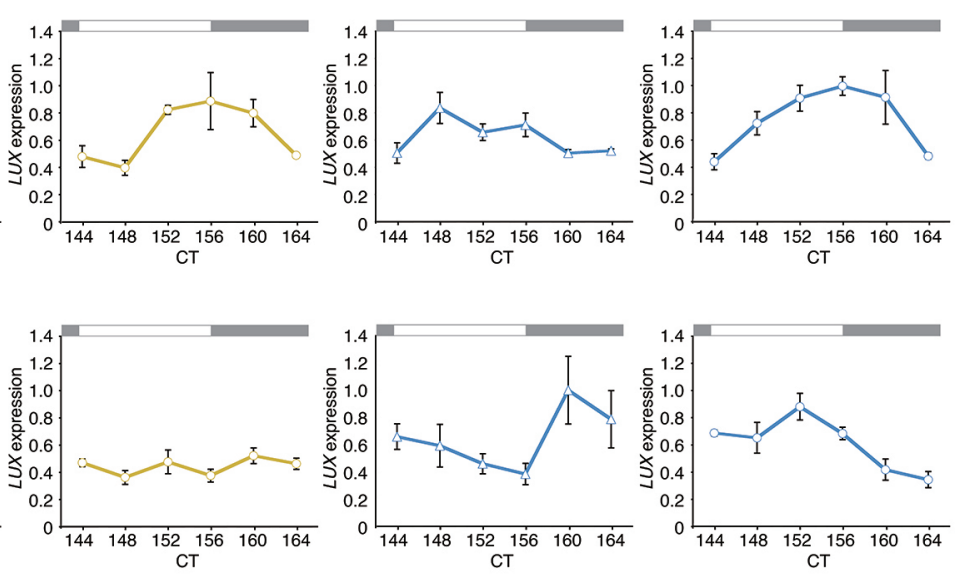

CER6::CCA1 \#8
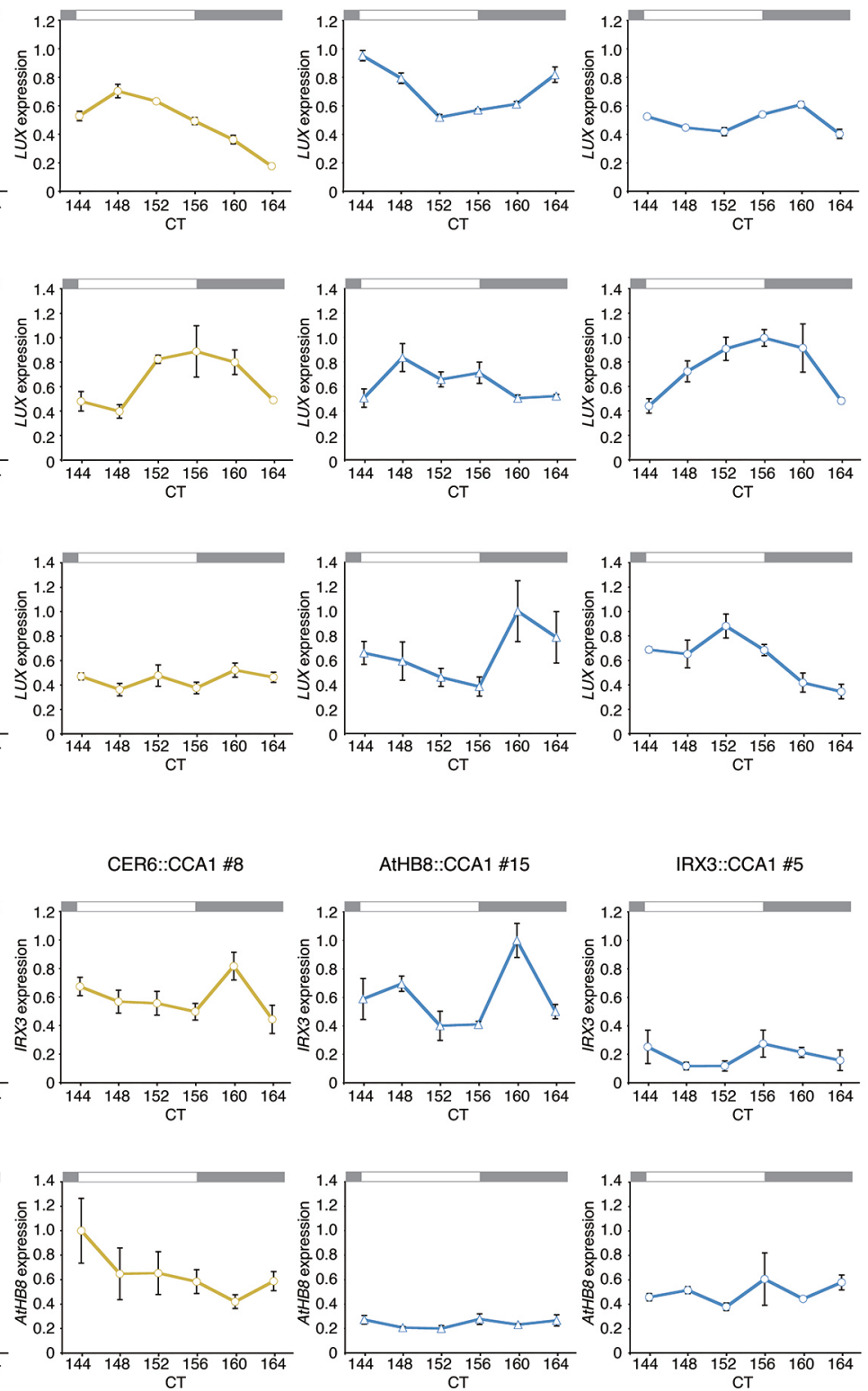

Supplementary Figure 3 I Diel expression patterns of LUX, IRX3, and AtHB8

a, Diel expression patterns of a clock gene, LUX ARRHYTHMO (LUX, also known as PCL1) in a specific tissue of each CCA1-GFP transgenic line. Plants were grown under L/D for 5 days and then transferred into continuous light conditions for 6 days. Seedlings were separated into mesophyll, vasculature, and epidermis every $4 \mathrm{~h}$ on day 12 . Endogenous $L U X$ expression was measured by qPCR and the geometric mean of APA1 and IPP2 was used as a control (error bars, s.e.m.; $\mathbf{n}=3$ ). The highest values are set as 1. $\mathbf{b}$, Diel expression patterns of IRX3 and AtHB8 as circadian clock regulated xylem- and (pro)cambium-specific genes, respectively. Plants were grown under L/D for 5 days and then transferred into continuous light conditions for 6 days, and whole cotyledons were collected every $4 \mathrm{~h}$. Gene expression levels were measured by qPCR and the geometric mean of APA1 and IPP2 was used as a control (error bars, s.e.m.; $n=3$ ). CT, circadian time. The highest values are set as 1 . 
a
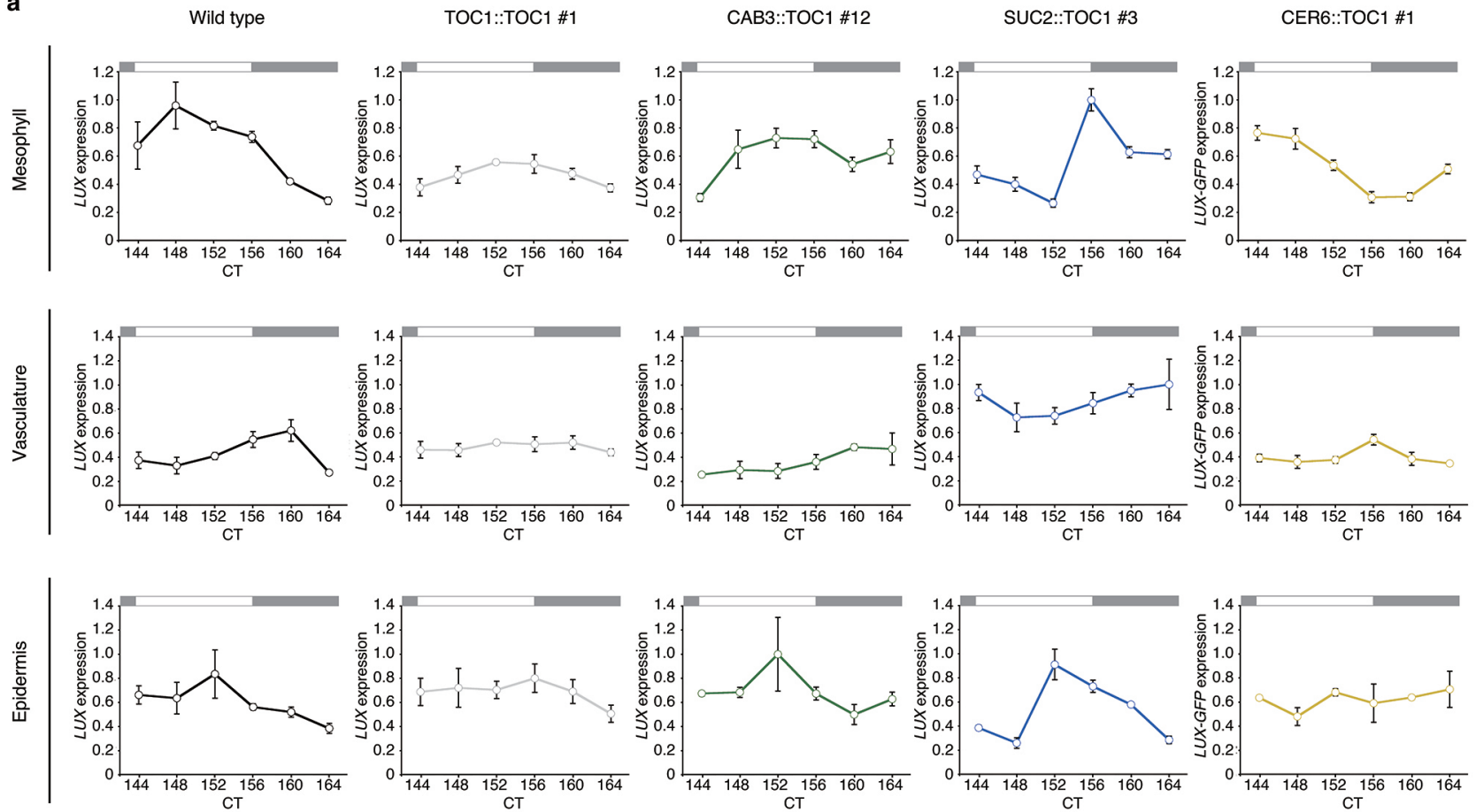

b

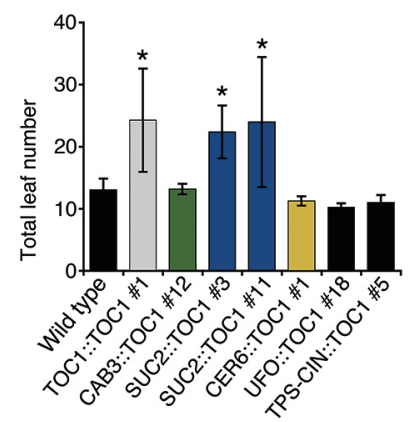

Supplementary Figure 4 I Effect of tissue-specifically expressed TOC1 for flowering time

a, Diel expression patterns of LUX in a specific tissue of each TOC1-GFP transgenic line. Plants were grown under L/D for 5 days and then transferred into continuous light conditions for 6 days. Seedlings were separated into mesophyll, vasculature, and epidermis every $4 \mathrm{~h}$ on day 12 . Endogenous $L U X$ expression was measured by qPCR and the geometric mean of APA1 and IPP2 was used as a control (error bars, s.e.m.; $\mathrm{n}=3$ ). CT, circadian time. The highest values are set as 1. b, Total leaf number at flowering for TOC1::TOC1 and SUC2::TOC1 were increased compared with wild type at $22^{\circ} \mathrm{C}$ under long-day (error bars, s.d.; $\mathrm{n}=12$ ). Significant difference from the corresponding wild types at ${ }^{*} \mathrm{P}<0.001$ by Dunnett's test. 

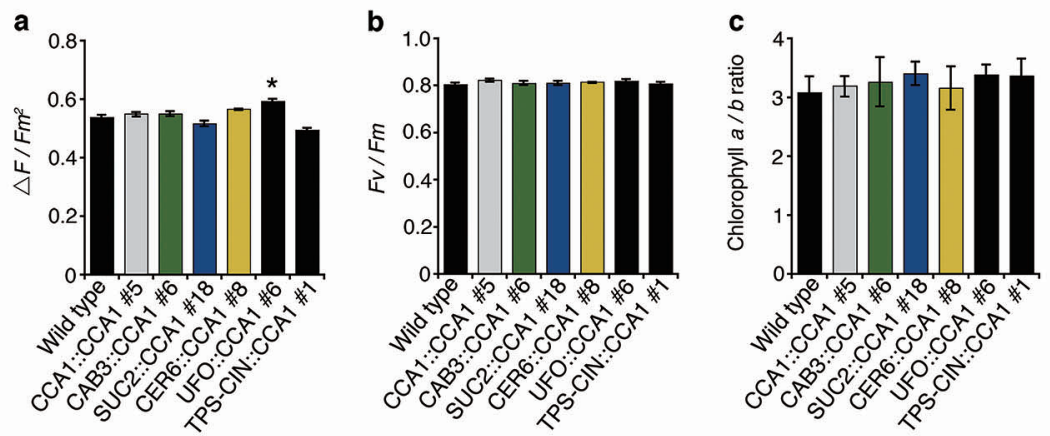

Supplementary Figure 5 I Effect of circadian clock in each tissue on the $\Delta F / F m$ ' (Yield), Fv/Fm and chlorophyll $\mathbf{a} / \mathrm{b}$ ratio

a, b, Plants were grown for 3 weeks at $22^{\circ} \mathrm{C}$ under long-day and $\Delta \mathrm{F} / \mathrm{Fm}$ ' (Yield) (a) and Fv/Fm (b) were measured with a pulse amplitude modulated (PAM) fluorometer (error bars, s.e.m.; $n=3$ ). c, Chlorophyll alb ratio of 10-day-old plants grown at $22^{\circ} \mathrm{C}$ under long-day (error bars, s.e.m.; $n=4$ ). Significant difference from the corresponding wild types at ${ }^{*} P<0.001$ by Dunnett's test. 

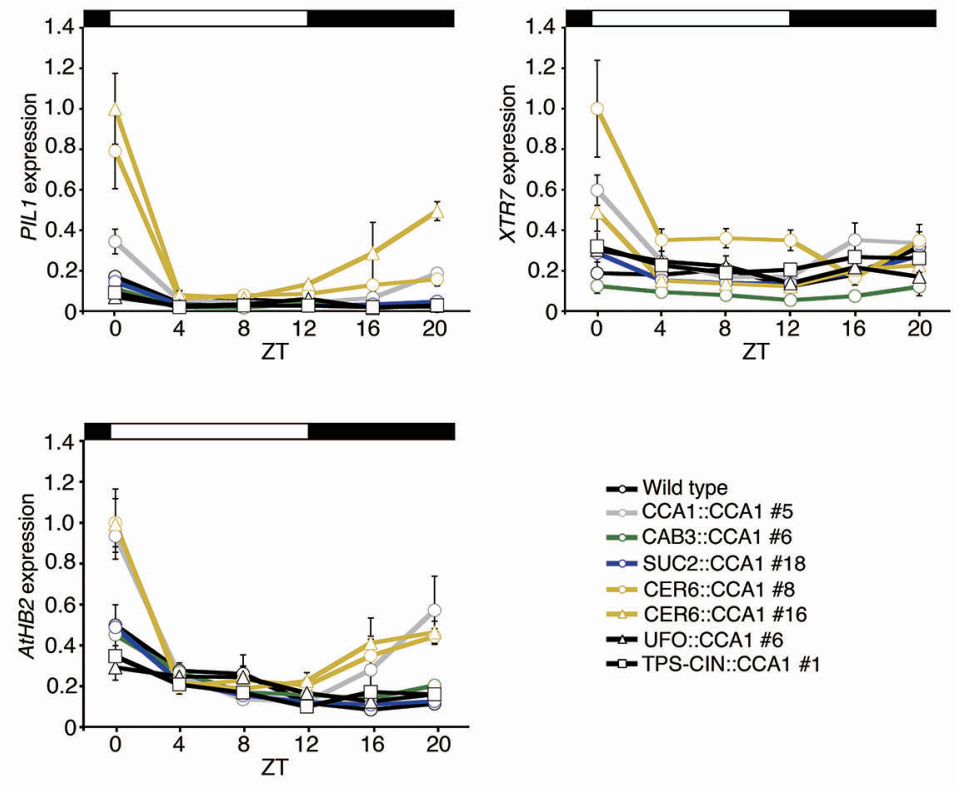

$\rightarrow$ Wild type $=$ CCA1 $:$ CCA 1 \#5 $=\mathrm{CAB} 3: \mathrm{CCA} 1$ \#6 $\sim$ SUC2::CCA1 \#18 $=0$ CER6::CCA1 \#8 $\Rightarrow=$ CER6::CCA1 \#16 $\rightarrow=$ UFO::CCA1 \#6 $=0-$ TPS-CIN::CCA1 \#1

\section{Supplementary Figure 6 I Diel expression of PIL1, XTR7, and AtHB2}

Diel expression of PIL1 (top left), XTR7 (top right), and AtHB2 (bottom left). 7-day-old seedlings grown at $22^{\circ} \mathrm{C}$ under L/D were analyzed every four hours by qPCR (error bars, s.e.m.; $n=3$ ). 
a
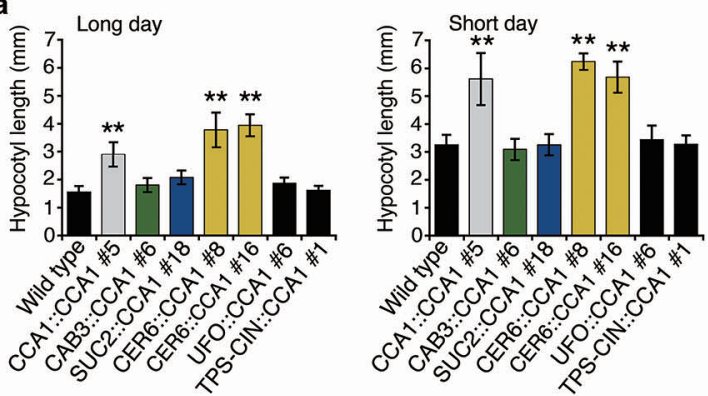

b
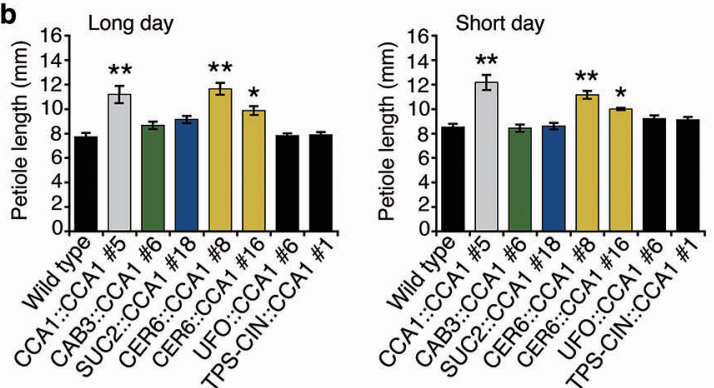

Supplementary Figure 7 I Hypocotyl and petiole lengths under long-day and short-day

a, Hypocotyl length of 7-day-old seedlings grown at $22^{\circ} \mathrm{C}$ under long-day and short-day (error bars, $S D ; n=20$ ). b, Petiole length of 5 and 6 th rosette leaves grown under long-day and short-day for 28 days (error bars, s.d.; $n$ $=10$ ). Significant difference from the corresponding wild types at ${ }^{\star} P<0.05$ and ${ }^{\star \star} P<0.01$ by Dunnett's test. 


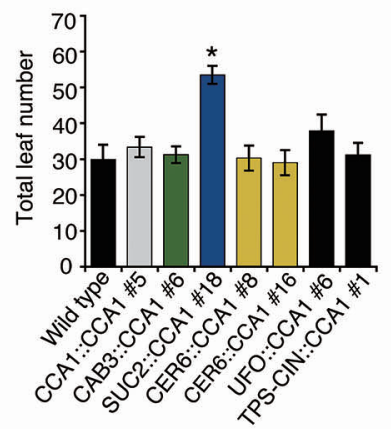

Supplementary Figure $8 \mathrm{I}$ Flowering time at $16^{\circ} \mathrm{C}$ under long-day Total leaf number at flowering was not affected in CCA1::CCA1 and SUC2::CCA1s at $16^{\circ} \mathrm{C}$ compared to $22^{\circ} \mathrm{C}$ (Fig. 1b) (error bars, s.d.; $n=12$ ). 


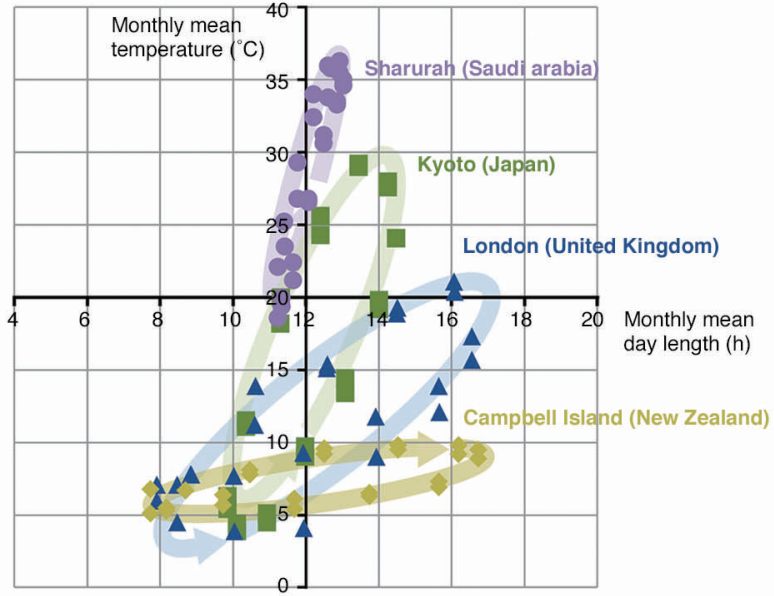

Supplementary Figure 9 I Dynamic varieties of temperature - photoperiod cycles on earth Interaction between temperature and photoperiod in Sharurah (Saudi Arabia). Kyoto (Japan), London (United Kingdom), and Campbell Island (New Zealand). The x-axis is the monthly mean temperature and the $y$-axis is the monthly mean photoperiod. Data from November 2012 to October 2014 are plotted. There is a wide range of variation in temperature - photoperiod relationships on earth. 